\title{
Effect of Roux-en-Y gastric bypass on the bioavailability of metoprolol from immediate and controlled release tablets: a single oral dose study before and after surgery
}

\author{
Jan Peter Yska, ${ }^{1}$ Jacquelien T M Wanders, ${ }^{1}$ Blessing Odigie, ${ }^{1}$ Jan A Apers, ${ }^{2}$ \\ Marloes Emous, ${ }^{2}$ Erik R E Totté, ${ }^{2}$ E Christiaan Boerma, ${ }^{3}$ Froukje L Ubels, ${ }^{4}$ \\ Herman J Woerdenbag, ${ }^{5}$ Henderik W Frijlink, ${ }^{5}$ Bob Wilffert, ${ }^{6,7}$ Eric N van Roon ${ }^{1,6}$
}

\begin{abstract}
- Additional material is published online only. To view please visit the journal online (http://dx.doi.org/ 10.1136/ejhpharm-2018001804).
\end{abstract}

For numbered affiliations see end of article.

\section{Correspondence to} Dr Jan Peter Yska, Clinical Pharmacy and Clinical Pharmacology, Medisch Centrum Leeuwarden, Leeuwarden 8901 BR, The Netherlands; j.p.yska@znb.nl, jpyska@upcmail.nl

Received 10 November 2018 Revised 12 January 2019 Accepted 17 January 2019 Published Online First 15 February 2019

EAHP Statement 4: Clinical Pharmacy Services.

\begin{abstract}
Objective Roux-en-Y gastric bypass (RYGB) surgery induces major changes in the gastrointestinal tract that may alter the pharmacokinetics of orally administered drugs. Results from pharmacokinetic studies are sparse. This study aimed to investigate the effect of RYGB on the bioavailability of metoprolol from immediate release (IR) and controlled release (CR) tablets in female patient volunteers before and after surgery.

Methods An explorative, two-phase, single oral dose pharmacokinetic study of metoprolol in female patients undergoing RYGB was carried out. The dose was administered twice in each patient, 1 month before and 6 months after surgery. After intake of either $100 \mathrm{mg}$ of metoprolol IR or CR tablet serum concentration-time profiles of metoprolol were determined. The endpoint was the ratio of $A U C_{\text {after }} / A U C_{\text {before }}$ of metoprolol.

Results Twelve patients were included in the study (metoprolol IR: 7; metoprolol CR: 5). After intake of a metoprolol IR tablet major intraindividual and interindividual differences for area under the serum concentration versus time curve (AUC) of metoprolol before and after surgery were observed (range ratio $\left.\mathrm{AUC}_{0-10 \text { hours after }} / A \cup C_{0-10 \text { hours before }}: 0.74-1.98\right)$. For metoprolol CR tablets a significant reduction in bioavailability of metoprolol was observed after surgery (range ratio $A \cup C_{0-24 \text { hours after }} / A \cup C_{0-24 \text { hours before }}: 0.43-0.77$ ). Conclusion RYGB may influence the bioavailability of metoprolol from an IR tablet. The magnitude of changes in bioavailability after RYGB requires close monitoring of patients using metoprolol IR tablets and dose adjustment if deemed necessary. RYGB clearly reduces the bioavailability of metoprolol from a CR tablet. After RYGB clinicians may consider to increase the dose according to clinical response.
\end{abstract}

\section{INTRODUCTION}

In 2014 Roux-en-Y gastric bypass (RYGB) was the second most commonly performed bariatric procedure in the world. ${ }^{1}$ RYGB achieves weight loss through restriction of food intake, altered neurohormonal signalling in body weight regulation, increased energy expenditure and altered bile salt metabolism due to changes in the gut microbiome. True malabsorption is rare after RYGB, but changes in anatomy and function such as a reduced gastric volume, increased gastric $\mathrm{pH}$, altered gastric emptying, reduced intestinal surface area for absorption, changed intestinal and hepatic first-pass metabolism, shorter intestinal transit time and more distal delivery of bile and pancreatic secretions may all affect the absorption and thus the bioavailability of orally administered drugs. ${ }^{2-4}$ After RYGB the performance of oral formulation types such as slow release or delayed release may suffer from critical changes affecting adequate drug absorption and thereby their efficacy. ${ }^{5}$

Generally after restrictive-malabsorptive procedures, such as RYGB, it is recommended to substitute controlled release (CR) drug formulations into immediate release (IR) dosage forms. ${ }^{6}$ This is reflected in the European guidelines on metabolic and bariatric surgery, stating that in the follow-up after bariatric surgery, patients should be advised to preferably use crushed and/or rapid release medication. ${ }^{7}$ However, these recommendations are not evidence based. So far, only a few studies have been published on the influence of RYGB on the pharmacokinetics of drugs, showing large variations. Moreover, changes are characterised by high unpredictability. ${ }^{8}$ Therefore, more studies are needed on the influence of RYGB on the pharmacokinetic behaviour. ${ }^{9}$

Bariatric surgery reduces the risk factors for cardiovascular disease. This influences the use of medication. After restrictive-malabsorptive weight loss procedures, such as RYGB, a significant reduction in use of beta-blockers has been reported, nonetheless a considerable number of patients still use a beta-blocker. ${ }^{10}{ }^{11}$ Metoprolol, a lipophilic cardioselective $\beta 1$-adrenoreceptor antagonist, has a long history of use in the treatment of hypertension, angina pectoris and other cardiovascular diseases, as well as for the prophylactic treatment of migraine. It is available as IR and CR tablet. After oral administration, absorption of metoprolol from the gastrointestinal tract is almost complete. Due to first-pass hepatic metabolism, about 50\% of the dose reaches the systemic circulation. In the liver metoprolol is metabolised for 70\%-80\% by cytochrome P450 2D6 (CYP2D6) into several metabolites. Alpha-hydroxymetoprolol is an active metabolite, possessing only around one-tenth of the $\beta 1$-blocking activity of metoprolol. It is formed by CYP2D6, making it a suitable marker for CYP2D6 activity. $^{12} 13$ In urine approximately $7 \%$ of the 
administered dose of metoprolol is recovered as $\alpha$-hydroxymetoprolol. Serum concentrations of $\alpha$-hydroxymetoprolol vary depending on age and on the oxidation phenotype. ${ }^{13} 14$

The aim of the present study was to investigate the effect of RYGB on the bioavailability of metoprolol and formation of its metabolite $\alpha$-hydroxymetoprolol, after a single oral dose of metoprolol IR and CR tablet in female bariatric surgery patient volunteers before and after surgery.

\section{METHODS}

\section{Participants}

The study was conducted in female patients undergoing RYGB. Only female patients were enrolled in this study, since for metoprolol gender-related differences exist in the pharmacokinetics leading to greater drug exposure (higher $\mathrm{C}_{\max }$ and area under the serum concentration versus time curve (AUC)) for women than men. ${ }^{15}$ Moreover, almost $80 \%$ of the patients undergoing bariatric surgery is female. ${ }^{16}$ The criteria for enrolment in this study were: female, age between 18 and 50 years, non-smokers, physiologically normal liver and kidney function, a normal ECG and extensive or intermediate CYP2D6 metaboliser status, as evidenced by genotyping. Pregnant patients, patients who had previously undergone gastrointestinal surgery and patients currently receiving metoprolol or with contraindications to treatment with metoprolol were excluded. Patients suffering from dumping syndrome after surgery were also excluded. After having given written informed consent the subjects underwent a medical examination by an internal medicine specialist. Genotyping was performed using TaqMan real-time PCR (LightCycler 480, Roche, Basel, Switzerland) and translated to a CYP2D6 predicted phenotype. ${ }^{17}$ Only patients meeting all criteria participated in the study, seven in the metoprolol IR and five in the metoprolol CR part. All subjects underwent RYGB surgery resulting in the creation of a gastric pouch with a volume of $20-70 \mathrm{~mL}$, a biliopancreatic limb of $80-150 \mathrm{~cm}$ and an alimentary limb of $150 \mathrm{~cm}$. In this study, subjects were their own control, taking the same tablet formulation of metoprolol before and after RYGB. Patients were allowed to participate in the IR and CR part of the study with an interval of at least 7 days between administrations of the two formulations of metoprolol.

\section{Metoprolol}

In the present study the bioavailability of metoprolol from two different oral tablet formulations before and after RYGB was investigated. Metoprolol tartrate $100 \mathrm{mg}$ IR tablet (Pharmachemie, Haarlem, The Netherlands (metoprolol IR)), and metoprolol succinate $95 \mathrm{mg}$ CR tablet, equivalent to $100 \mathrm{mg}$ of metoprolol tartrate (Pharmachemie (metoprolol CR)) were used.

\section{Study design}

An explorative, two-phase single oral dose pharmacokinetic study of metoprolol was performed. A metoprolol IR or CR tablet was administered twice to each patient, 1 month before and 6 months after surgery. After an overnight fast of at least 10 hours subjects came to the clinical research unit of the hospital. Usual medication of the patients was adjusted in a way that interference with the study was prevented and that the patient's treatment was guaranteed. After insertion of a peripheral intravenous cannula participants ingested a metoprolol IR or CR tablet with $150 \mathrm{~mL}$ water (presurgery), or with as little water as possible (postsurgery). Blood samples were collected at $0,0.5,1,1.5$ (only IR), 2, 3, 4, 5, 6, 8, 10 and 24 (only CR) hours after intake. After the 10 hours blood sample was taken, subjects were allowed to go home. For subjects taking metoprolol CR a 24-hour blood sample was taken at the patient's home. Within 2 hours after collection blood samples were centrifuged for $10 \mathrm{~min}$ at $2000 \mathrm{~g}$ at $4^{\circ} \mathrm{C}$ and serum samples were stored at $-24^{\circ} \mathrm{C}$ until analysis. A standardised snack and standardised meals were served 2, 4 and 10 hours after administration of the tablet, respectively. During the first 6 hours after intake of the medication the patients were not allowed to lie down. For safety reasons blood pressure and heart rate were regularly monitored. Concentrations of metoprolol and its metabolite $\alpha$-hydroxymetoprolol were determined by means of a validated LC-Orbitrap MS method. ${ }^{18}$ The lower limit of quantitation of the assay was $2.0 \mathrm{ng} / \mathrm{mL}$ for metoprolol and $1.0 \mathrm{ng} / \mathrm{mL}$ for $\alpha$-hydroxymetoprolol, respectively. Four months after surgery, before the start of the second phase of the study, the patients were asked about dumping syndrome symptoms by means of a questionnaire. ${ }^{19}$ Complications of the surgery which might interfere with the study were also assessed. The subject was withdrawn from the study if she was suffering from the dumping syndrome or from interfering complications.

\section{Pharmacokinetic parameters}

The following pharmacokinetic parameters of metoprolol and $\alpha$-hydroxymetoprolol were determined: maximum concentration $\left(\mathrm{C}_{\max }\right)$, time to maximum concentration $\left(\mathrm{T}_{\max }\right)$ (metoprolol IR only) and AUC for 10 (metoprolol IR) or 24 hours (metoprolol CR). The areas under the serum concentration versus time curves $\left(\mathrm{AUC}_{0-10 \text { hours }}\right.$ or $\mathrm{AUC}_{0-24 \text { hours }}$ ) were determined using the linear trapezoidal rule in Microsoft Excel (2013). The endpoint was the ratio $\mathrm{AUC}_{\text {after }} / \mathrm{AUC}_{\text {before }}$ of metoprolol and $\alpha$-hydroxymetoprolol for each participant. A paired t-test was performed on the obtained data before and after surgery. A p value $<0.05$ was considered significant.

\section{RESULTS}

Seven patients were included in the metoprolol IR part of the study, five patients in the metoprolol CR part. Two patients participated in both studies.

In table 1 the characteristics of the participants of the pharmacokinetic study are shown. After intake of the single dose of metoprolol no side effects were reported. After surgery no complications from the gastric bypass surgery which might interfere with the study occurred and the participants did not suffer from symptoms of the dumping syndrome. No subjects were withdrawn from the study.

In table 2 pharmacokinetic parameters after intake of metoprolol IR and CR, respectively, before and after RYGB are

\section{Table 1 Characteristics of study participants}

\begin{tabular}{|c|c|c|}
\hline Characteristic & $\begin{array}{l}\text { Metoprolol immediate } \\
\text { release tablet }\end{array}$ & $\begin{array}{l}\text { Metoprolol controlled } \\
\text { release tablet }\end{array}$ \\
\hline Participants (n) & 7 & 5 \\
\hline Age, mean $\pm S D$ (year) & $37 \pm 8$ & $37 \pm 5$ \\
\hline $\begin{array}{l}\text { Extensive CYP2D6 } \\
\text { metaboliser }(n)\end{array}$ & 2 & 1 \\
\hline $\begin{array}{l}\text { Intermediate CYP2D6 } \\
\text { metaboliser ( } \mathrm{n})\end{array}$ & 5 & 4 \\
\hline \multicolumn{3}{|l|}{$\mathrm{BMI}$, mean $\pm \mathrm{SD}\left(\mathrm{kg} / \mathrm{m}^{2}\right)$} \\
\hline Before surgery & $39.9 \pm 2.1$ & $38.6 \pm 4.0$ \\
\hline $5-6$ months after surgery & $30.8 \pm 2.3$ & $29.2 \pm 4.3$ \\
\hline$-\Delta$ & $9.1 \pm 1.2^{*}$ & $9.4 \pm 0.7^{*}$ \\
\hline
\end{tabular}

${ }^{*} \mathrm{P}<0.01$

$\mathrm{BMI}$, body mass index 
Table 2 Pharmacokinetic parameters after administration of a single oral dose of metoprolol tartrate 100 immediate release (IR) tablet (seven participants) and metoprolol succinate $95 \mathrm{mg}$ controlled release (CR) tablet (five participants)

\begin{tabular}{|c|c|c|c|c|c|c|}
\hline \multirow[b]{2}{*}{ Parameter } & \multicolumn{3}{|c|}{ Metoprolol } & \multicolumn{3}{|c|}{$\alpha-\mathrm{OH}$ metoprolol } \\
\hline & Mean \pm SD & Range & $P$ value & Mean \pm SD & Range & $P$ value \\
\hline \multicolumn{7}{|l|}{ Metoprolol IR } \\
\hline \multicolumn{7}{|c|}{$A U C_{0-10 \text { hours }}(\mathrm{ng} \cdot$ hour $/ \mathrm{mL})$} \\
\hline Pre-RYGB & $391 \pm 144$ & $197-569$ & & $394 \pm 60$ & $322-493$ & \\
\hline Post-RYGB & $446 \pm 168$ & $165-670$ & 0.24 & $477 \pm 92$ & $339-619$ & 0.01 \\
\hline Ratio & $1.19 \pm 0.43$ & $0.74-1.98$ & & $1.21 \pm 0.14$ & $1.05-1.36$ & \\
\hline \multicolumn{7}{|l|}{$C_{\max }(\mathrm{ng} / \mathrm{mL})$} \\
\hline Pre-RYGB & $96 \pm 33$ & 64-156 & & $62 \pm 11$ & $49-76$ & \\
\hline Post-RYGB & $119 \pm 53$ & 43-204 & 0.30 & $77 \pm 15$ & $51-90$ & 0.01 \\
\hline \multicolumn{7}{|l|}{$\mathrm{T}_{\max }$ (hour) } \\
\hline Pre-RYGB & $1.4 \pm 0.9$ & $0.5-3.0$ & & $1.8 \pm 1.0$ & $0.5-3.1$ & \\
\hline Post-RYGB & $1.0 \pm 0.3$ & $0.5-1.5$ & 0.42 & $1.3 \pm 0.4$ & $0.9-2.0$ & 0.18 \\
\hline \multicolumn{7}{|c|}{ Metoprolol CR } \\
\hline \multicolumn{7}{|c|}{$\mathrm{AUC}_{0-24 \text { hours }}(\mathrm{ng} \cdot \mathrm{hour} / \mathrm{mL})$} \\
\hline Pre-RYGB & $361 \pm 159$ & $200-548$ & & $337 \pm 123$ & $203-529$ & \\
\hline Post-RYGB & $225 \pm 137$ & $105-421$ & $<0.01$ & $300 \pm 81$ & $182-390$ & 0.22 \\
\hline Ratio & $0.59 \pm 0.13$ & $0.43-0.77$ & & $0.91 \pm 0.10$ & $0.74-0.98$ & \\
\hline
\end{tabular}

AUC, area under the serum concentration versus time curve; RYGB, Roux-en-Y gastric bypass.

presented. Online supplementary appendix figures A1 and A2 display the individual serum concentration-time profiles of metoprolol and $\alpha$-hydroxymetoprolol after administration of either a single dose of metoprolol IR or CR, respectively, before and after RYGB. Figure 1 shows the change in AUC of metoprolol and $\alpha$-hydroxymetoprolol for individual participants, after administration of metoprolol IR and CR, respectively, before and after RYGB.

After intake of metoprolol IR tablet metoprolol exposure, $\mathrm{C}_{\max }$ and $\mathrm{T}_{\max }$ were not significantly altered by RYGB. However, large intraindividual and interindividual differences for AUC of metoprolol (range ratio $\mathrm{AUC}_{\text {after }} / \mathrm{AUC}_{\text {before }}: 0.74-1.98$ ), as well as for $\mathrm{C}_{\max }$ and $\mathrm{T}_{\max }$ were observed before and after surgery. The metabolite $\alpha$-hydroxymetoprolol had a greater, significant exposure after RYGB $(\mathrm{p}=0.01)$. In $\mathrm{C}_{\max }$ a significant increase was also seen $(p=0.01)$.

After intake of metoprolol CR tablet a significant reduction in bioavailability of metoprolol was observed $(\mathrm{p}<0.01$; range ratio $\left.\mathrm{AUC}_{\text {after }} / \mathrm{AUC}_{\text {before }}: 0.43-0.77\right)$. For $\alpha$-hydroxymetoprolol the difference in exposure was not significant, with ratios of $\mathrm{AUC}_{\text {after }}$ $\mathrm{AUC}_{\text {before }}$ ranging from 0.74 to 0.98 .
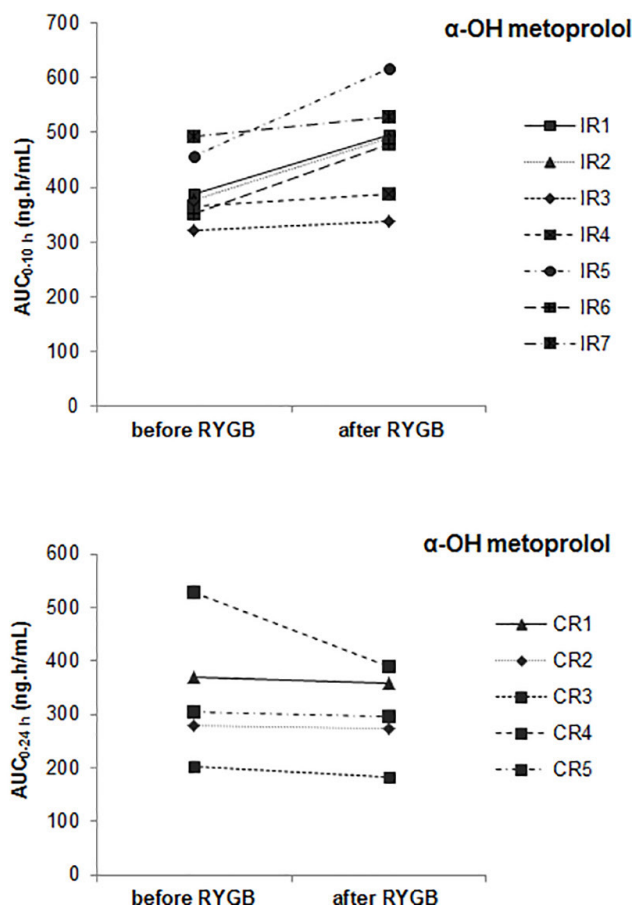

Figure $1 \mathrm{AUC}_{0-10 \text { hours }}$ after administration of a single oral dose of metoprolol tartrate $100 \mathrm{mg}$ immediate release (IR) tablet (upper windows) and AUC 24 hours after administration of a single oral dose of metoprolol succinate $95 \mathrm{mg}$ controlled release (CR) tablet (lower windows) before and after Roux-en-Y gastric bypass (RYGB). AUC, area under the serum concentration versus time curve. 
For both metoprolol IR and CR extensive metabolisers for CYP2D6 had an AUC for metoprolol in the lower range, before as well as after surgery.

\section{DISCUSSION}

This study found that RYGB may influence the bioavailability of metoprolol from IR tablets. Large interindividual differences in the ratios of $\mathrm{AUC}_{\text {after }} / \mathrm{AUC}_{\text {before }}$ of metoprolol were observed. After intake of an IR tablet exposure of metoprolol after RYGB may be reduced or increased. For the individual patient the consequences of RYGB for dosing metoprolol IR tablets cannot be predicted. The magnitude of changes in bioavailability after RYGB requires close monitoring of patients using metoprolol IR tablets and dose adjustment if deemed necessary.

The bioavailability of metoprolol from CR tablets is reduced after RYGB. Therefore, after RYGB clinicians may consider to increase the dose according to clinical response.

Several factors that might affect bioavailability of metoprolol after RYGB should be considered. Metoprolol has been classified as a Biopharmaceutics Classification Scheme class I substance, having a high solubility and a high intestinal permeability, meaning that metoprolol will be easily dissolved and absorbed. ${ }^{20}$ Metoprolol is not absorbed from the stomach, but may be well and similarly absorbed throughout the small intestine and colon. Thus, metoprolol administered as an oral formulation is expected to be absorbed from any region of the intestine in which it is released. ${ }^{21-23}$ After RYGB the duodenum and proximal jejunum are no longer available for absorption, so reduced absorption may occur. In obese patients metoprolol, being a liposoluble compound, was found to have a higher volume of distribution with a lower $\mathrm{C}_{\max }$ compared with non-obese patients. ${ }^{24}$ With a decreased body mass index and body fat percentage after RYGB, one might expect a higher bioavailability.

Before enrolment in the study, participants were genotyped for CYP2D6 and poor metabolisers were excluded from participation. More intermediate than extensive metabolisers were included. This may be coincidence and due to the small numbers of inclusion. Although after RYGB after intake of a metoprolol IR tablet exposure of metoprolol was either reduced or increased for $\alpha$-hydroxymetoprolol a significant increase in AUC was observed. (Patho)physiological alterations associated with obesity may have a specific impact on drug metabolism and elimination. Brill et $a l^{25}$ found that in literature few studies indicate trends towards increased CYP2D6-mediated metabolites in obese versus non-obese patients. However, Abdussalam et al ${ }^{26}$ showed that in rats given a high-fat diet CYP2D1 (the equivalent in rat to human CYP2D6) is downregulated. Higher levels of $\alpha$-hydroxymetoprolol after RYGB might be caused by increased CYP2D6 metabolism due to weight loss. After intake of a metoprolol CR tablet bioavailability of metoprolol was reduced, but, except for one patient, no reduction in AUC was observed for $\alpha$-hydroxymetoprolol after RYGB. Lower exposure of metoprolol after RYGB did not lead to lower levels of $\alpha$-hydroxymetoprolol. This might be caused by increased CYP2D6 metabolism due to weight loss after RYGB.

As might be expected, extensive CYP2D6 metabolisers participating in this study showed a bioavailability of metoprolol in the lower range before as well as after RYGB. However, after intake of a metoprolol IR tablet, a decrease as well as an increase in bioavailability after RYGB was observed, depending on the subject.

In the present study after RYGB decreased bioavailability of metoprolol from CR tablet is observed. In normal patients systemic availability of CR metoprolol formulations is 20\%-30\% lower than that after administration of an IR tablet. ${ }^{14}$ According to Plosker and Clissold, ${ }^{14}$ the reduction in bioavailability is likely related to the relatively slow rate of drug delivery which enhances presystemic clearance of metoprolol because hepatic extraction is a saturable process. Moreover, since transit time through the gastrointestinal tract can vary, some active drug may remain in the CR preparations at the time they are eliminated from the body. This might also contribute to reduced systemic availability in some patients. After RYGB, intestinal transit time might be a limiting factor for absorption from a CR formulation. However, data on intestinal transit time after RYGB are sparse and conflicting. Using a sulfasalazine tablet as a marker, Carswell et $a l^{27}$ found no influence of RYGB on orocaecal transit time. Dirksen $e a^{28}$ showed that 1.5 years after RYGB pouch emptying time was shorter after a meal, but small intestinal transit was slower in patients than in control subjects. Colonic transit time did not differ. In patients 5.7 years after RYGB Nguyen et al ${ }^{29}$ found that pouch emptying and caecal arrival time were more rapid compared with control subjects. Pouch emptying tended to be faster after $150 \mathrm{~mL}$ than after $50 \mathrm{~mL}$ drinks. In bioavailability studies oral tablet formulations are usually ingested with $150 \mathrm{~mL}$ of water. Because we did not want pouch emptying be influenced by the ingested volume of water, we decided to administer the metoprolol IR and CR tablets with $150 \mathrm{~mL}$ of water before surgery and with as little water as possible after surgery.

Possible variations in absorption of metoprolol after RYGB might be caused by changes in release from the oral tablet formulations. In vitro dissolution experiments showed that release patterns of metoprolol from IR and CR tablets in simulated RYGB conditions did not differ from those found in conditions before RYGB. Under all conditions applied, metoprolol IR and CR tablets showed adequate dissolution, fully complying with pharmacopoeial requirements. ${ }^{30}$ From this, we conclude that the observed changes in bioavailability of metoprolol after RYGB are not caused by changes in release rate of metoprolol from the studied IR and CR tablet formulations as a result of environmental conditions.

As yet, for some drugs the influence of RYGB on pharmacokinetic properties has been evaluated in controlled clinical studies. However, well-designed clinical studies with repeated measures before and after surgery are scarce and not all effects of RYGB on drug exposure reported so far are of clinical importance. ${ }^{8931}$ An example of a well-designed study is the repeated measure study by Mitrov-Winkelmolen et al. ${ }^{32}$ They found a faster absorption of both acetylsalicylic acid and omeprazole after RYGB with a higher exposure of acetylsalicylic acid and an average decrease in bioavailability of omeprazole. Their findings have consequences for the dosing of omeprazole after RYGB (increase the dose in patients with inadequate response), but not for acetylsalicylic acid.

Gesquiere et $a l^{33}$ performed a single-dose pharmacokinetic study of metoprolol tartrate $200 \mathrm{mg}$ IR and CR formulations in 14 healthy volunteers before and 6-8 months after RYGB. They concluded that the oral exposure of metoprolol from immediate and controlled-release formulations was not significantly different after RYGB compared with before, although there was a tendency towards higher exposure after surgery. This different outcome compared with the results from our study may be explained in terms of different design in the Gesquiere et al's study. Women (10) as well as men (4) were included. Before inclusion the CYP2D6 genotype of the patients was not determined. In addition, no individual data were presented, only mean $\mathrm{AUC}_{0-24 \text { hours }}$ with 95\% CI. Serum concentrations 
of metabolites of metoprolol were not measured. After RYGB participants ingested the tablets with $150 \mathrm{~mL}$ of water. By drinking this volume, pouch emptying might be accelerated. ${ }^{29}$ Moreover, in daily practice patients may not swallow a tablet with such a volume of water.

This study has several limitations. The sample sizes of the metoprolol IR and CR part of the study were small. The findings of this study may have implications for dosing metoprolol after RYGB, despite the fact that this was an explorative pharmacokinetic study. Only women were included in this study. Although pharmacokinetics of metoprolol is different in women compared with men, the effects of RYGB on bioavailability of metoprolol after oral administration in men might be comparable. After surgery this study was performed 6 months after RYGB. Relatively soon after surgery or in the long term results might have been different. After RYGB intestinal adaptation may occur, where mucosal hypertrophy within the remaining intestine gives an increase in absorptive capacity over time. It is unknown, however, whether intestinal adaptation affects absorption. ${ }^{4}$ Pharmacodynamics and pharmacokinetics of metoprolol are stereospecific. Metoprolol is used as a racemate. However, the assay used in this study was not enantioselective for metoprolol. Generally, beta-blockers are absorbed from the gastrointestinal tract via passive diffusion. Therefore, their absorption is not considered stereoselective. ${ }^{34}$

\section{CONCLUSIONS}

RYGB may influence the bioavailability of metoprolol from an IR tablet. After RYGB clinicians may consider to adjust the dose according to clinical response. RYGB reduces the bioavailability of metoprolol from a CR tablet. After RYGB clinicians may consider to increase the dose according to clinical response.

\section{What this paper adds}

\section{What is already known on this subject}

- Roux-en-Y gastric bypass (RYGB) induces major changes in the gastrointestinal tract that may alter the pharmacokinetics of orally administered drugs.

- Results from pharmacokinetic studies are sparse.

What this study adds

- After RYGB possible changes in bioavailability of metoprolol from immediate release tablets require close monitoring of patients, with dose adjustment if necessary.

- Because of reduced bioavailability of metoprolol from controlled release tablets after RYGB, the dose may be increased, according to clinical response.

\section{Author affiliations}

'Department of Clinical Pharmacy and Clinical Pharmacology, Medisch Centrum Leeuwarden, Leeuwarden, The Netherlands

${ }^{2}$ Department of Surgery, Medisch Centrum Leeuwarden, Leeuwarden, The Netherlands

${ }^{3}$ Department of Intensive Care, Medisch Centrum Leeuwarden, Leeuwarden, The Netherlands

${ }^{4}$ Department of Internal Medicine, Medisch Centrum Leeuwarden, Leeuwarden, The Netherlands

${ }^{5}$ Department of Pharmaceutical Technology and Biopharmacy, University of Groningen, Groningen, The Netherlands

${ }^{6}$ Unit of Pharmacotherapy, Pharmacoepidemiology and Pharmacoeconomics, Groningen Research Institute of Pharmacy, University of Groningen, Groningen, The Netherlands

${ }^{7}$ Department of Clinical Pharmacy and Pharmacology, University Medical Center, University of Groningen, Groningen, The Netherlands
Acknowledgements We thank Berend Oosterhuis, PharmD, PhDt, for his fruitful discussions and ideas for setting up the study, and Daan J Touw, PharmD, PhD (Department of Clinical Pharmacy and Pharmacology, University Medical Center Groningen, University of Groningen, Groningen, The Netherlands) for his review of the results of the study. († deceased)

Contributors Study concept and design: JPY, ENvR, JTMW, BW, HWF, HJW. Acquisition of data: JPY, BO, JAA, ME, ERET, ECB, FLU. Analysis and interpretation of data: JPY, ENvR, BW, HWF, HJW. Drafting of the manuscript: JPY. Critical revision of the manuscript for important intellectual content: all authors. Study supervision: JPY, ENvR

Funding This study was funded by a grant from the Science Fund (Wetenschapsfonds) Medical Centre Leeuwarden.

Competing interests None declared.

Patient consent for publication Not required.

Ethics approval The regional research ethics committee reviewed and approved this study with EudraCT numbers 2013-002260-10 and 2013-002274-41.

Provenance and peer review Not commissioned; externally peer reviewed.

\section{REFERENCES}

1 Angrisani L, Santonicola A, lovino P, et al. Bariatric surgery and endoluminal procedures: IFSO worldwide survey 2014. Obes Surg 2017;27:2279-89.

2 De Smet J, Van Bocxlaer J, Boussery K. The influence of bypass procedures and other anatomical changes in the gastrointestinal tract on the oral bioavailability of drugs. J Clin Pharmacol 2013:53:361-76.

3 Smith A, Henriksen B, Cohen A. Pharmacokinetic considerations in Roux-en-Y gastric bypass patients. [correction appears in Am J Health Syst Pharm 2011;68:2241-7]. Am J Health Syst Pharm 2012;69:182.

4 Padwal R, Brocks D, Sharma AM. A systematic review of drug absorption following bariatric surgery and its theoretical implications. Obes Rev 2010;11:41-50.

5 Lizer $\mathrm{MH}$, Papageorgeon $\mathrm{H}$, Glembot TM. Nutritional and pharmacologic challenges in the bariatric surgery patient. Obes Surg 2010;20:1654-9.

6 Miller AD, Smith KM. Medication and nutrient administration considerations after bariatric surgery. Am J Health Syst Pharm 2006;63:1852-7.

7 Fried M, Yumuk V, Oppert JM, et al. Interdisciplinary European guidelines on metabolic and bariatric surgery. Obes Surg 2014;24:42-55.

8 Hachon L, Declèves $X$, Faucher $P$, et al. RYGB and drug disposition: how to do better? Analysis of pharmacokinetic studies and recommendations for clinical practice. Obes Surg 2017;27:1076-90

9 Yska JP, van der Linde S, Tapper VV, et al. Influence of bariatric surgery on the use and pharmacokinetics of some major drug classes. Obes Surg 2013;23:819-25.

10 Malone M, Alger-Mayer SA. Medication use patterns after gastric bypass surgery for weight management. Ann Pharmacother 2005;39:637-42.

11 Yska JP, van der Meer DH, Dreijer AR, et al. Influence of bariatric surgery on the use of medication. Eur J Clin Pharmacol 2016;72:203-9.

12 Murthy SS, Shetty HU, Nelson WL, et al. Enantioselective and diastereoselective aspects of the oxidative metabolism of metoprolol. Biochem Pharmacol 1990;40:1637-44.

13 McGourty JC, Silas JH, Lennard MS, et al. Metoprolol metabolism and debrisoquine oxidation polymorphism-population and family studies. Br J Clin Pharmacol 1985:20:555-66.

14 Plosker GL, Clissold SP. Controlled release metoprolol formulations. A review of their pharmacodynamic and pharmacokinetic properties, and therapeutic use in hypertension and ischaemic heart disease. Drugs 1992;43:382-414.

15 Luzier AB, Killian A, Wilton JH, et al. Gender-related effects on metoprolol pharmacokinetics and pharmacodynamics in healthy volunteers. Clin Pharmacol Ther 1999:66:594-601.

16 Chang SH, Stoll CR, Song J, et al. The effectiveness and risks of bariatric surgery: an updated systematic review and meta-analysis, 2003-2012. JAMA Surg 2014:149:275-87.

17 KNMP. General background text pharmacogenetics - CYP2D6. 2016. https://www. knmp.nl/downloads/g-standaard/farmacogenetica/english-background-information/ cyp2d6-english.pdf (Accessed 24 Jun 2017).

18 Postma-Kunnen S, Yska JP, Hommema G, et al. A validated high-resolution accurate mass LC-MS assay for quantitative determination of metoprolol and $\alpha$-hydroxymetoprolol in human serum for application in pharmacokinetics. J App/ Bioanal 2017;3:49-57.

19 Laurenius A, Olbers T, Näslund I, et al. Dumping syndrome following gastric bypass: validation of the dumping symptom rating scale. Obes Surg 2013;23:740-55.

20 Food and Drug Administration, Center for Drug Evaluation and Research (CDER). Guidance for industry: Waiver of in vivo bioavailability and bioequivalence studies for immediate-release solid oral dosage forms based on a biopharmaceutics classification system. 2017. https://www.fda.gov/downloads/Drugs/Guidances/ucm070246.pdf (Accessed 4 Jan 2018). 


\section{Original research}

21 Jobin G, Cortot A, Godbillon J, et al. Investigation of drug absorption from the gastrointestinal tract of man. I. Metoprolol in the stomach, duodenum and jejunum. Br J Clin Pharmacol 1985;19:975-105.

22 Vidon N, Evard D, Godbillon J, et al. Investigation of drug absorption from the gastrointestinal tract of man. II. Metoprolol in the jejunum and ileum. Br J Clin Pharmacol 1985;19:1075-12.

23 Godbillon J, Evard D, Vidon N, et al. Investigation of drug absorption from the gastrointestinal tract of man. III. Metoprolol in the colon. Br J Clin Pharmacol 1985;19:113S-8.

24 Galletti F, Fasano ML, Ferrara LA, et al. Obesity and beta-blockers: influence of body fat on their kinetics and cardiovascular effects. J Clin Pharmacol 1989;29:212-6.

25 Brill MJ, Diepstraten J, van Rongen A, et al. Impact of obesity on drug metabolism and elimination in adults and children. Clin Pharmacokinet 2012;51:277-304.

26 Abdussalam A, Al-Agili M, Al Nebaihi HM, et al. Dietary-induced obesity and changes in the biodistribution and metabolism of amiodarone in the rat. J Pharm Sci 2018;107:2938-45.

27 Carswell KA, Vincent RP, Belgaumkar AP, et al. The effect of bariatric surgery on intestinal absorption and transit time. Obes Surg 2014;24:796-805.
28 Dirksen C, Damgaard M, Bojsen-Møller KN, et al. Fast pouch emptying, delayed small intestinal transit, and exaggerated gut hormone responses after Roux-en-Y gastric bypass. Neurogastroenterol Motil 2013;25:346-e255.

29 Nguyen NQ, Debreceni TL, Burgstad CM, et al. Effects of posture and meal volume on gastric emptying, intestinal transit, oral glucose tolerance, blood pressure and gastrointestinal symptoms after Roux-en-Y gastric bypass. Obes Surg 2015;25:1392-400

30 Yska JP, Punter RJ, Woerdenbag HJ, et al. A gastrointestinal simulation system for dissolution of oral solid dosage forms before and after Roux-en-Y gastric bypass. Eur $J$ Hosp Pharm 2019;26:152-6.

31 Greenblatt HK, Greenblatt DJ. Altered drug disposition following bariatric surgery: a research challenge. Clin Pharmacokinet 2015;54:573-9.

32 Mitrov-Winkelmolen L, van Buul-Gast MW, Swank DJ, et al. The effect of Roux-en-Y gastric bypass in morbidly obese patients on pharmacokinetics of (acetyl) salicylic acid and omeprazole: the ERY-PAO study. Obes Surg 2016:26:2051-8.

33 Gesquiere I, Darwich AS, Van der Schueren B, et al. Drug disposition and modelling before and after gastric bypass: immediate and controlled-release metoprolol formulations. Br J Clin Pharmacol 2015;80:1021-30.

34 Mehvar R, Brocks DR. Stereospecific pharmacokinetics and pharmacodynamics of beta-adrenergic blockers in humans. J Pharm Pharm Sci 2001;4:185-200. 